\title{
Identification of Key Pathways and Genes in SARS-CoV-2 Infecting Human Intestines by Bioinformatics Analysis
}

\author{
Ji-Chun Chen ${ }^{1} \cdot$ Tian-Ao Xie ${ }^{1} \cdot$ Zhen-Zong Lin $^{1} \cdot$ Yi-Qing Li ${ }^{1} \cdot$ Yu-Fei Xie ${ }^{1}$. \\ Zhong-Wei Li ${ }^{1} \cdot$ Xu-Guang Guo ${ }^{1,2,3,4}$ (D)
}

Received: 4 August 2021 / Accepted: 21 October 2021 / Published online: 17 November 2021

(c) The Author(s) 2021

\begin{abstract}
COVID-19 is a serious infectious disease that has recently swept the world, and research on its causative virus, SARS-CoV-2, remains insufficient. Therefore, this study uses bioinformatics analysis techniques to explore the human digestive tract diseases that may be caused by SARS-CoV-2 infection. The gene expression profile data set, numbered GSE149312, is from the Gene Expression Omnibus (GEO) database and is divided into a 24-h group and a 60-h group. $\mathrm{R}$ software is used to analyze and screen out differentially expressed genes (DEGs) and then gene ontology (GO) term and Kyoto Encyclopedia of Genes and Genomes (KEGG) pathway enrichment analyses are performed. In KEGG, the pathway of non-alcoholic fatty liver disease exists in both the 24-h group and 60-h group. STRING is used to establish a protein-protein interaction (PPI) network, and Cytoscape is then used to visualize the PPI and define the top 12 genes of the node as the hub genes. Through verification, nine statistically significant hub genes are identified: AKT1, TIMP1, NOTCH, CCNA2, RRM2, TTK, BUB1B, KIF20A, and PLK1. In conclusion, the results of this study can provide a certain direction and basis for follow-up studies of SARS-CoV-2 infection of the human digestive tract and provide new insights for the prevention and treatment of diseases caused by SARS-CoV-2.
\end{abstract}

Keywords SARS-CoV-2 $\cdot$ Bioinformatics

Xu-Guang Guo

gysygxg@gmail.com

1 Department of Clinical Medicine, The Third Clinical School of Guangzhou Medical University, Guangzhou 510150, China

2 Department of Clinical Laboratory Medicine, The Third Affiliated Hospital of Guangzhou Medical University, Guangzhou 510150, China

3 Key Laboratory for Major Obstetric Diseases of Guangdong Province, The Third Affiliated Hospital of Guangzhou Medical University, Guangzhou 510150, China

4 Key Laboratory of Reproduction and Genetics of Guangdong Higher Education Institutes, The Third Affiliated Hospital of Guangzhou Medical University, Guangzhou 510150, China 


\section{Introduction}

In December 2019, pneumonia caused by a new type of coronavirus appeared in the world. The World Health Organization (WHO) initially named it COVID-19 (coronavirus disease 2019). Since then, the Coronavirus Research Group of the International Commission on Virology has officially named the virus severe acute respiratory syndrome coronavirus type 2 (SARS-CoV-2) (Harapan et al. 2020).

SARS-CoV-2 is a positive-stranded RNA virus with an envelope. It is spherical under a transmission electron microscope, with a diameter ranging from 60 to $140 \mathrm{~nm}$ and a unique peak length of $8-12 \mathrm{~nm}$. It belongs to the coronavirus $2 \mathrm{~b}$ lineage (Zhu et al. 2020; He et al. 2020). Studies have shown that SARS-CoV-2 infects host cells through the combination of spike protein and angiotensin-converting enzyme II (ACE2). It spreads faster and is highly stable, but it is not very lethal (He et al. 2020).

SARS-CoV-2 can spread through the respiratory tract, saliva, contact, and excrement; the possibility of transmission through aerosols is also high (Chan et al. 2020). COVID-19 lurks in the human body for about 6.4 days on average and anyone is susceptible to infection (Wang et al. 2020). Currently, the clinical symptoms of COVID-19 are mainly fever (90\% or even higher), cough (about 75\%), and breathing difficulties (up to 50\%) (Cipriano et al. 2020; Huang et al. 2020; Li et al. 2020). Some patients have symptoms of gastrointestinal diseases, such as nausea and vomiting. Studies have found that SARS-CoV-2 is present in the feces of COVID-19 patients, which suggest the possibility of SARS-CoV-2 infecting the human digestive tract and causing a series of diseases (Han et al. 2020). Furthermore, according to WHO statistics, as of July 27, 2020, there were a total of 15,785,641 cases of COVID-19 and 64,016 deaths worldwide. COVID19 has become a communicable disease that seriously endangers global public health. Therefore, this study aims to provide new ideas and methods for clinically diagnosing, treating, and limiting the spread of SARS-CoV-2 by studying how it infects the human digestive tract.

Bioinformatics is an essentially interdisciplinary field that uses aspects of computer science, mathematics, and statistics to store, manage, analyze, and interpret biological data (Jin et al. 2020). This study uses bioinformatics analysis to identify and analyze the differential expression of organisms under different conditions and then uses gene ontology (GO) analysis and Kyoto Encyclopedia of Genes and Genomes (KEGG) analysis to obtain the biological significance of DEGs and establish a visual protein-protein interaction (PPI) network, finally leading to a conclusion (Ostaszewski et al. 2020). 


\section{Methods and Materials}

\section{Data Collection}

We obtained the GSE149312 (Lamers et al. 2020) gene expression profile as microarray data from the GEO database (https://www.ncbi.nlm.nih.gov/geo/). In the original study, the authors treated intestinal organ samples with SARSCoV or SARS-CoV-2 for 24 or $60 \mathrm{~h}$, respectively. RNA was then extracted under dilation conditions (Exp) or differentiation conditions (DIF). We only took the SARS-COV-2 infection group and blank group from the original study, grouping them according to the infection time. In each group, there were 4 infected samples and 6 control samples.

\section{Differentially Expressed Genes (DEG) Screening}

To obtain the DEGs between the experimental group and the control group as well as the corresponding volcano map and heat map, $\mathrm{R}$ software (version 4.0.1) and pheatmap package were used in this analysis, and $P<0.05$ and $I \log 2$ fold change (FC) $\mid>1$ were set as the threshold for judging DEGs. (Xie et al. 2019 Jul) DEGs were considered to have statistical significance within this critical value. The online tool, Venny (version 2.1.0; https://bioinfogp.cnb.csic.es/tools/venny/), was then used to make the Venn diagram of the DEGs shared by the 24-h group and 60-h group. All R scripts have been reported to the GitHub repository. (https://github.com/authe ntic-zz/mycode.git).

\section{The Function and Pathway Enrichment Analysis of DEGs}

$\mathrm{R}$ language was used to conduct the GO analysis and KEGG pathway analysis to acquire the biological significance of DEGs. GO is a commonly used approach in bioinformatics analysis. Its function is to annotate genes and their products (Gene Ontology Consortium 2006; Liang et al. 2016). Methods to recognize gene function annotations include the biological process (BP), cell component (CC), and molecular function (MF) categories. KEGG is also a commonly used bioinformatics database for integrating and analyzing a large number of data sets obtained from highthroughput experimental technologies, such as genome sequencing (Liang et al. 2016; Kanehisa and Goto 2000). $P<0.05$ was considered significant.

\section{Visualization of the Establishment and Module Selection of PPI Networks and the Identification of the Hub Genes}

To evaluate the interrelationship between DEGs and build a visual protein interaction network, the STRING online database (version 11.0; http://string-db.org/) was used. First, the DEGs were entered into the STRING online database and the required minimum cross-evaluation was set to a medium confidence level $>0.4$. Second, because the initial PPI obtained in STRING was complicated, Cytoscape was 


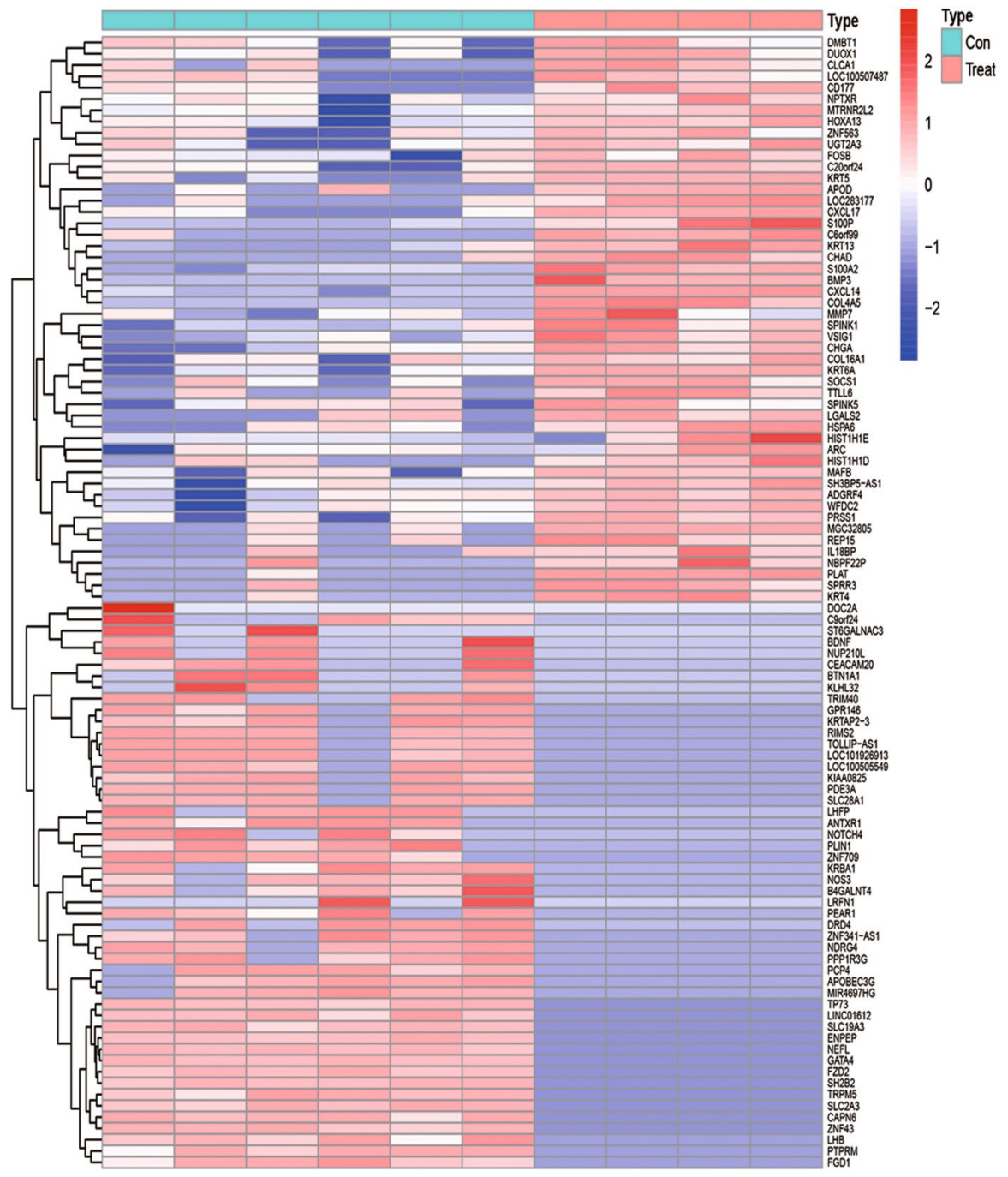

Fig. 1 Heat map of the DEGS from the 24-h group

used to build a visual PPI relationship network. The plug-in cytoHubba in Cytoscape was used to analyze the core gene modules of the PPI network complex (the default parameters) and define the top 12 genes of the node as the hub genes.

\section{Verification of the Hub Genes}

The 12 defined hub genes were uploaded to GraphPad Prism (version 8.0.2) in the order of high to low. Then GSE150728 was analyzed by bioinformatics, and the data 


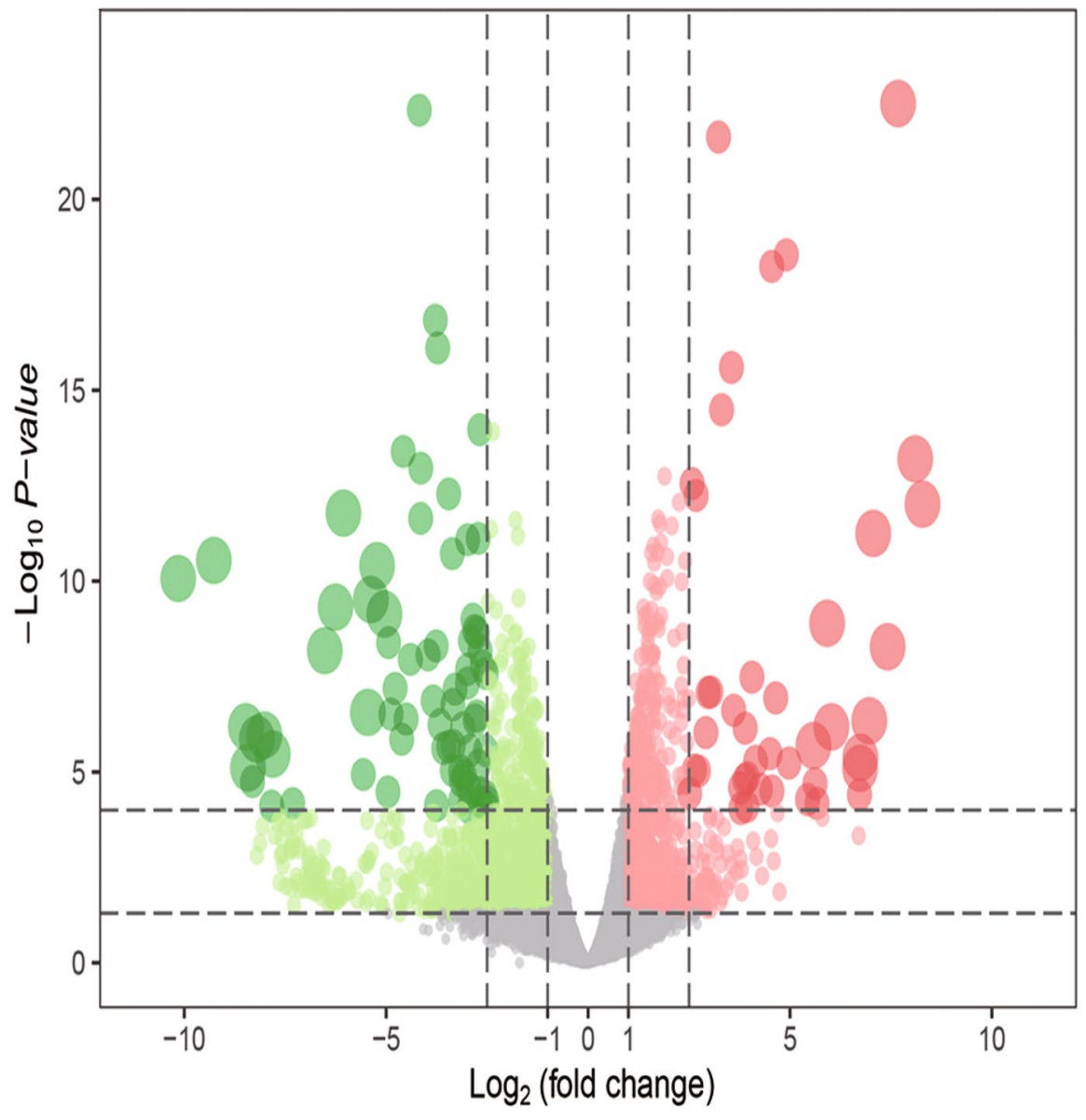

Fig. 2 Volcano plot of the DEGS from the 24-h group

obtained passed t test and non-parametric test and $P<0.05$ was used as the standard to screen for statistically significant hub genes.

\section{Results}

\section{The Identification of DEGs'}

According to the established criteria, 1930 DEGs were screened from the 24-h group, including 748 up-regulated genes and 1182 down-regulated genes. These DEGs are shown in the heat map (Fig. 1) and a volcano plot (Fig. 2) of the 24-h group. From the 60-h group, 1638 DEGs were screened, including 890 up-regulated genes and 748 down-regulated genes. These DEGs are shown in the heat map 


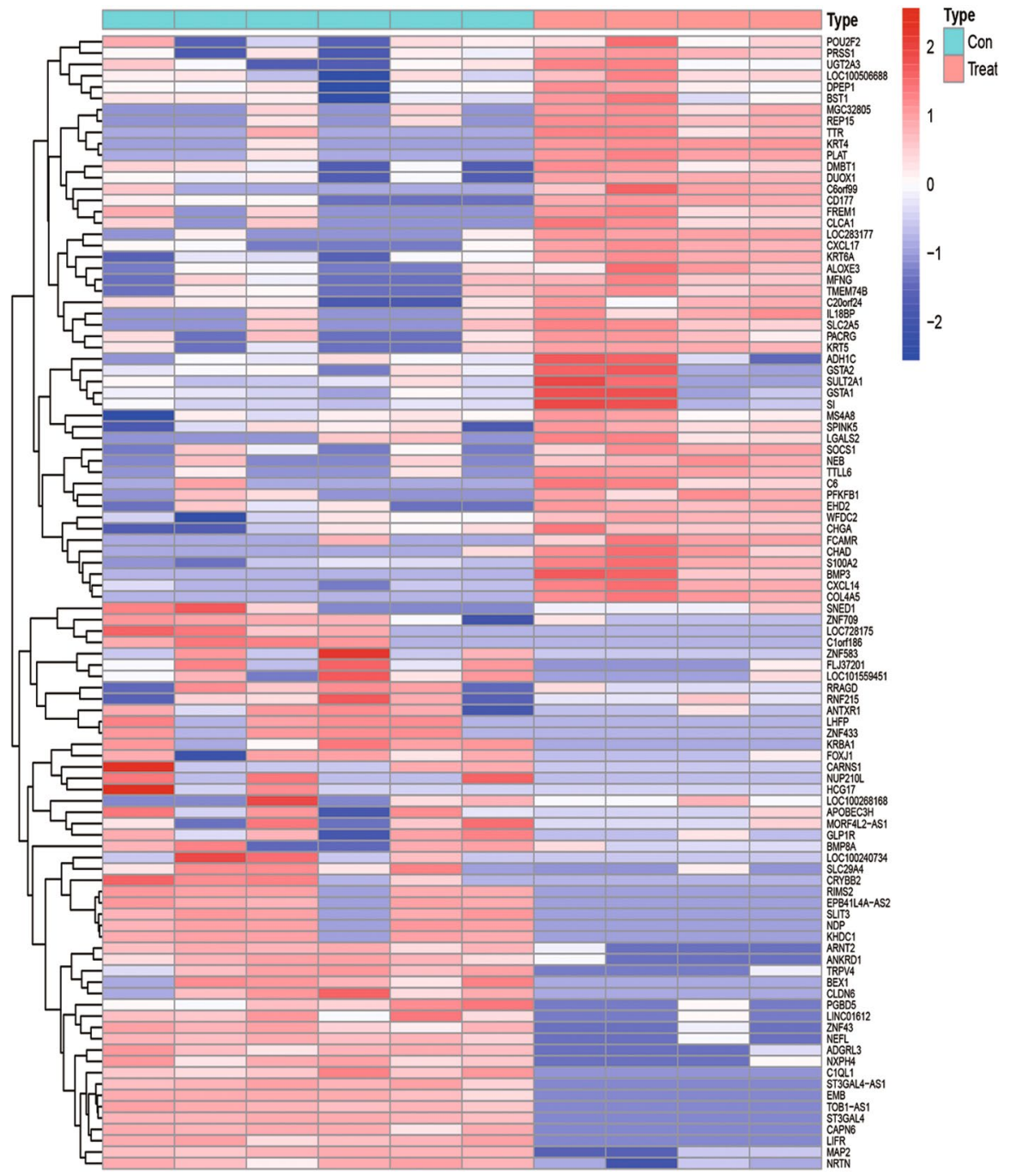

Fig. 3 Heat map of the DEGS from the 60-h group

(Fig. 3) and a volcano plot (Fig. 4) of the 60-h group. Through the analysis of the Venn diagram (Fig. 5), 744 DEGs co-expressed between the 24-h group and the 60-h group were obtained. More detailed data are shown in Table S1.

\section{GO Term Enrichment Analysis of DEGs}

The BP analysis showed that the DEGs of the 24-h group (Fig. 6) were significantly enriched in mitotic nuclear division, nuclear division, mitotic sister chromatid segregation, negative regulation of the cell cycle process, and chromosome segregation. 


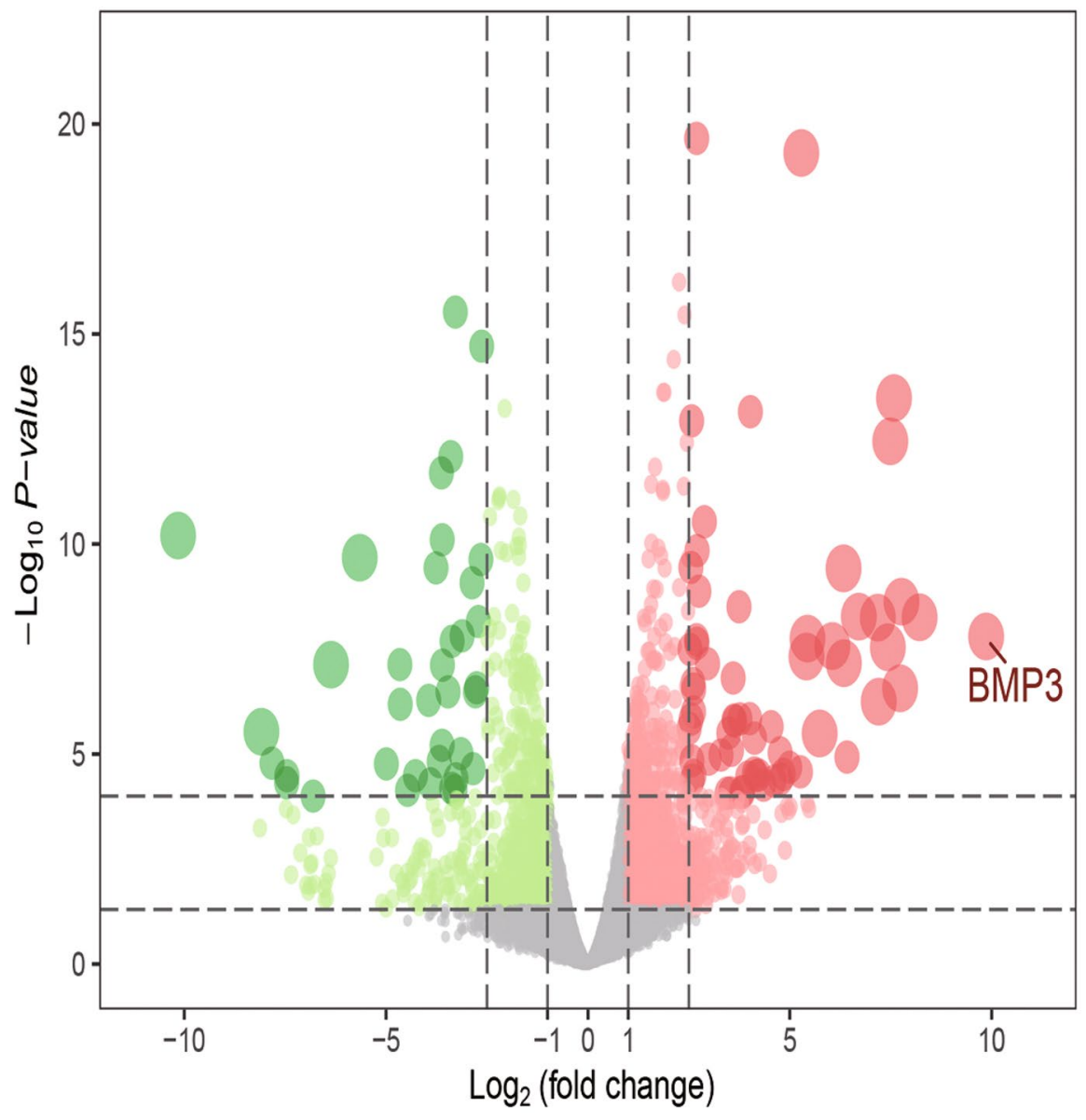

Fig. 4 Volcano plot of the DEGS from the 60-h group

The DEGs of the 60-h group (Fig. 7) were found in small molecule catabolic process and fatty acids and significantly enriched in acid metabolic process, long-chain fatty acid metabolic process, cellular response to xenobiotic stimulus, and unsaturated fatty acid metabolic process. Among them, there was no intersection between the 24-h group and the $60 \mathrm{~h}$ group. More detailed data are shown in Table S2.

\section{KEGG Pathway Analysis of DEGs}

In order to explore the potential mechanism of these DEGs, KEGG pathway analysis was performed using the R software. The results of KEGG analysis showed that the DEGs of 24-h group (Fig. 8) were significantly enriched in cell cycle, small cell lung cancer, DNA replication, cellular senescence, and non-alcoholic fatty liver disease. The DEGs of the 60-h group (Fig. 9) were found in chemical carcinogenesis and 


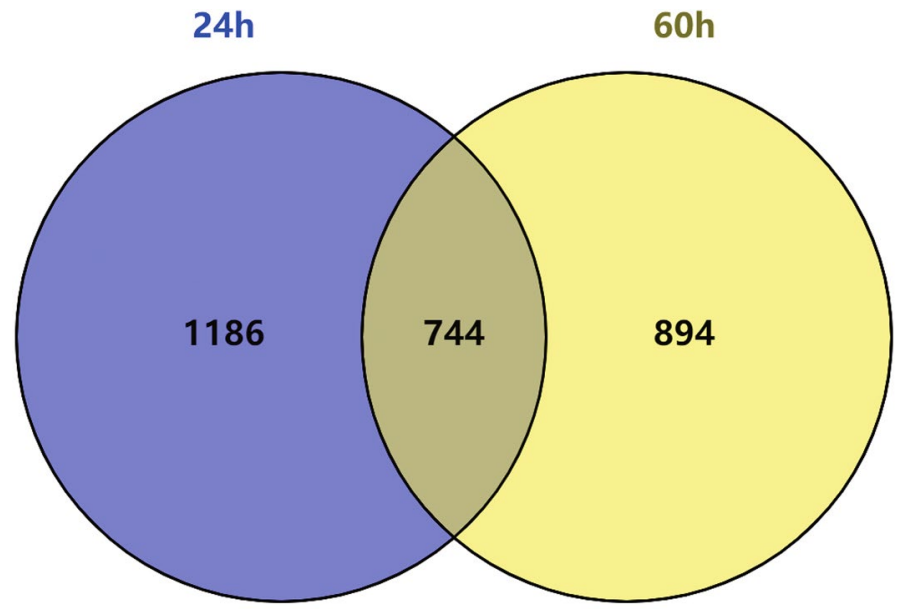

Fig. 5744 DEGs co-expressed between the 24-h group and the 60-h group

mineral absorption and significantly enriched in non-alcoholic fatty liver disease, drug metabolism-other enzymes, and metabolism of xenobiotics by cytochrome P450. Among them, non-alcoholic fatty liver disease was a pathway co-expressed in the 24-h group and 60-h group. More detailed data are shown in Table S3.

\section{Protein-Protein Interaction Network Analysis of DEGs}

Based on the STRING database, we constructed PPI networks of the DEGs in the 24-h group, 60-h group, and intersection group. The PPI network of the 24-h group (Fig. 10) included 1778 nodes and 1457 edges, the PPI network of the 60-h group (Fig. 11) included 710 nodes and 1457 edges, and the PPI network of the intersection group (Fig. 12) included 692 nodes and 162 edges. The top twelve genes (Fig. 13) were defined as the hub genes, namely AKT1, TIMP1, SERPINA1, NOTCH1, CCNA2, RRM2, TTK, RACGAP1, BUB1B, H2AFX, KIF20A, and PLK1.

\section{Verification of the Hub Genes}

Through $t$ tests and non-parametric tests, with the standard set as $P<0.05$, nine statistically significant genes (Fig. 14) were finally obtained, including AKT1, TIMP1, NOTCH1, CCNA2, RRM2, TTK, BUB1B, KIF20A, and PLK1. These genes may play a pivotal role in the impact of SARS-CoV-2 on the human intestine. 


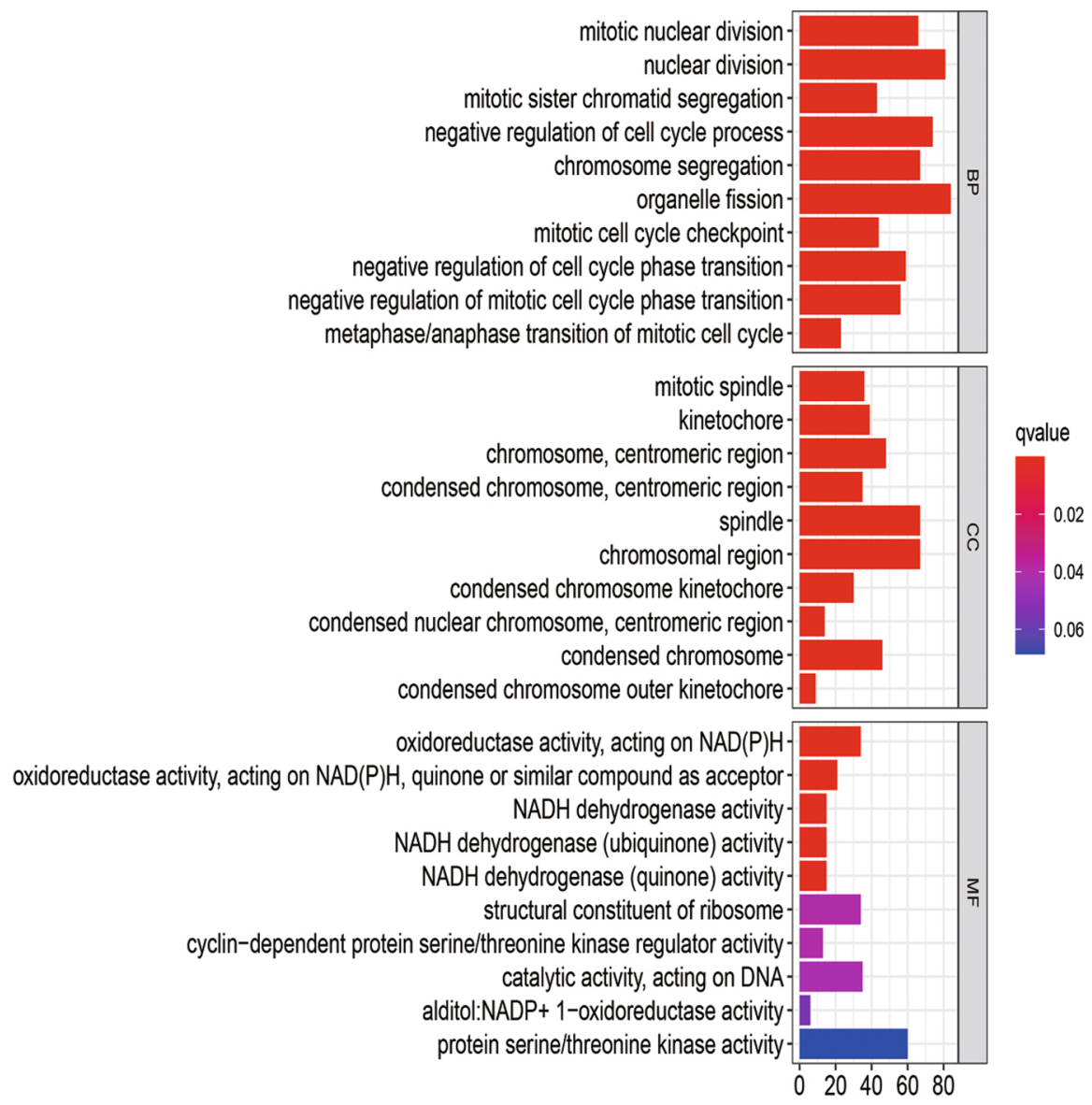

Fig. 6 GO analysis of the DEGS from the 24-h group

\section{Discussion}

Currently, many types of mutations have appeared in SARS-CoV-2, but vaccination has not yet been popularized as vaccines are still in clinical trials. In this study, we identified the DEGs between the SARS-CoV-2 and the normal samples. To understand these DEGs better, we conducted GO function and KEGG pathway analysis on them and constructed a PPI network to determine the hub genes.

Angiotensin-converting enzyme (ACE2) is thought to be the mechanism of SARS-CoV-2-infected cells. SARS-CoV-2 activates the intestinal ACE2 receptor, causing inflammation (intestinal inflammation) and eventually diarrhea (Villapol 2020). Existing studies have shown that the high expression of ACE2 is not limited to lung type II alveolar cells (AT2); they are also found in the gastrointestinal tract, especially in the absorptive intestinal epithelial cells of the ileum and colon (Hajifathalian et al. 2020), which provide a scientific basis for the detection of 


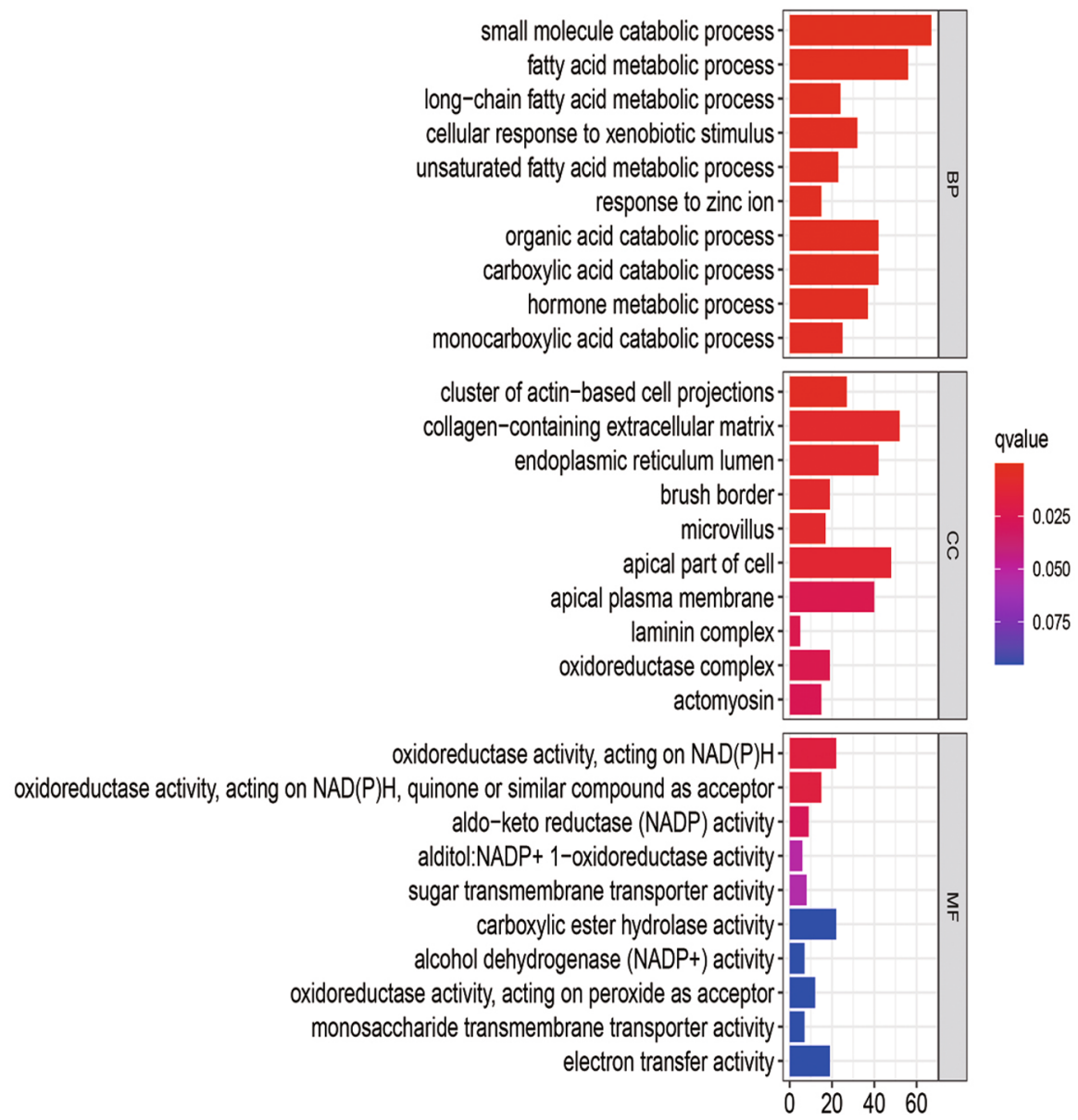

Fig. 7 GO analysis of the DEGS from the 60-h group

SARS-CoV-2 RNA in the stool samples of some patients, indicating that the digestive system is a potential route of COVID-19 infection (Cipriano et al. 2020). Moreover, in the results of the BP process screening in the 60-h GO functional annotation group, we found that the cells' response to heterologous biological stimuli was significantly enriched. In previous studies, we found that following exposure to the initial stimulus, innate immune cells will experience metabolic, mitochondrial, and epigenetic reprogramming, leading to an immune response with an enhanced memory phenotype (Geller and Yan 2020).

In the analysis of the KEGG pathway, we found that the significant pathways in the 24-h group and 60-h group had a cross-path: non-alcoholic fatty liver disease. Intestinal dysfunction can cause changes in intestinal microbes and increase inflammatory cytokines, leading to the aggravation of symptoms and even more serious complications (Villapol 2020). An increase in the rate of abnormal liver function 


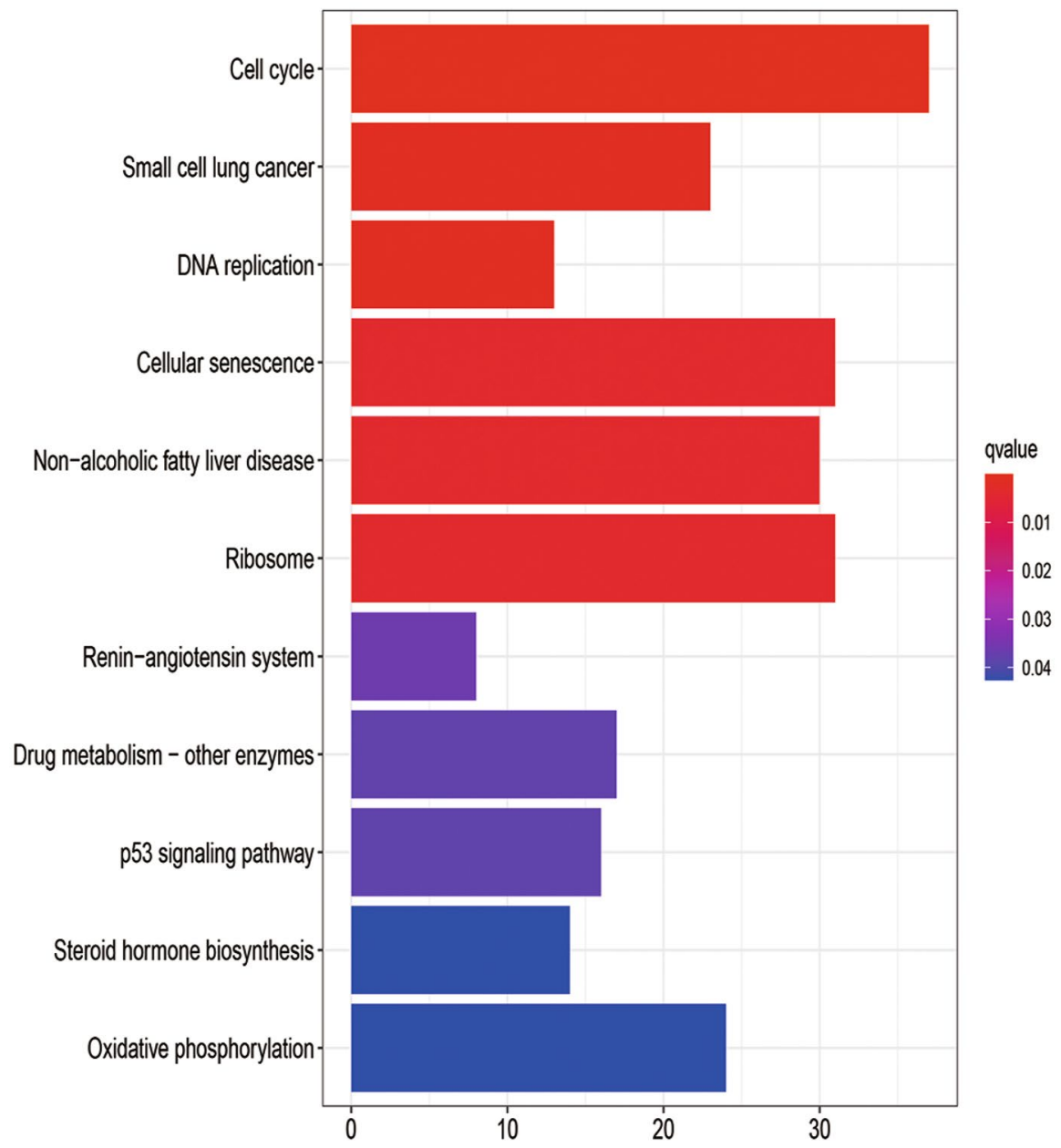

Fig. 8 KEGG pathway enrichment analysis of DEGs from the 24-h group

has been observed in patients with severe COVID-19. The microbiota can aggravate NAFLD through certain mechanisms, including changing the permeability of the intestines and the energy absorbed by the diet (Safari and Gérard 2019), so NAFLD patients may also be more susceptible to the increased cytokine production associated with COVID-19 (Prins and Olinga 2020). Studies have suggested that patients with NAFLD may be particularly vulnerable to SARS-CoV-2 infection and complications resulting from COVID-19 (Portincasa et al. 2020).

In GO analysis and KEGG pathway analysis, DEGs were found to be enriched in cell cycle. Coronavirus $\mathrm{N}$ protein is located in the cytoplasm and is involved in virus replication and assembly. Previous reports have indicated that the expression of the coronavirus $\mathrm{N}$ protein may affect the cell cycle (Zuwała et al. 2015). Both SARS and COVID-19 are considered to be pandemic infectious diseases caused by 


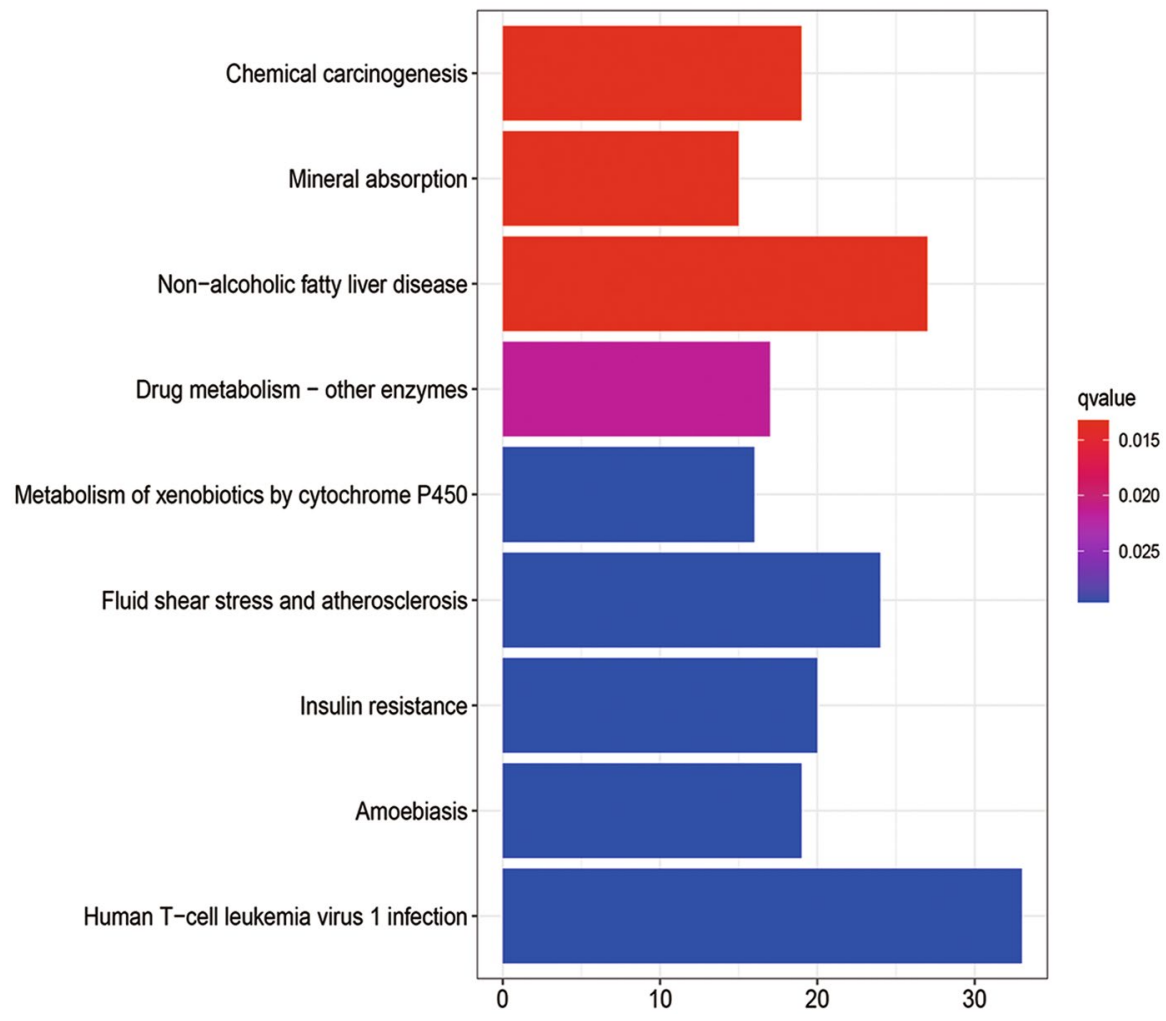

Fig. 9 KEGG pathway enrichment analysis of DEGs from the 60-h group

coronaviruses, which show that cell cycle research can play a significant role in their prevention and control.

Among the hub genes, the expression of TIMP1, CCNA2, RRM2, TTK, BUB1B, PLK1, and KIF20A were up-regulated, while the expression of ATK1 and NOTCH1 were down-regulated.

AKT1 is one of the subtypes of AKT. The down-regulation of AKT1 expression will promote the M1 polarization of macrophages, and M1 macrophages will secrete high levels of cytokines that can cause inflammation (Arranz et al. 2012). TIMP1 is a member of the tissue inhibitor of the metalloproteinase family (TIMP). As the coronavirus infection progresses, the expression of TIMP will increase correspondingly, and it is clearly expressed in lymphocytes, macrophages, and eosinophils during inflammation (Zhou et al. 2005). It can induce colon cell carcinogenesis through the FAK-PI3K/AKT and MAPK pathways (Song et al. 2016). The ATK1 and TIMP1 genes have a certain stimulating effect on immune cells, such as macrophages, and trigger the immune mechanism of the immune system. This indicates that SARS-CoV-2 may cause severe inflammatory bowel disease, fever, diarrhea, and other symptoms after infecting the human body, eventually causing damage to the human gastrointestinal system. Studies have shown that the overexpression 


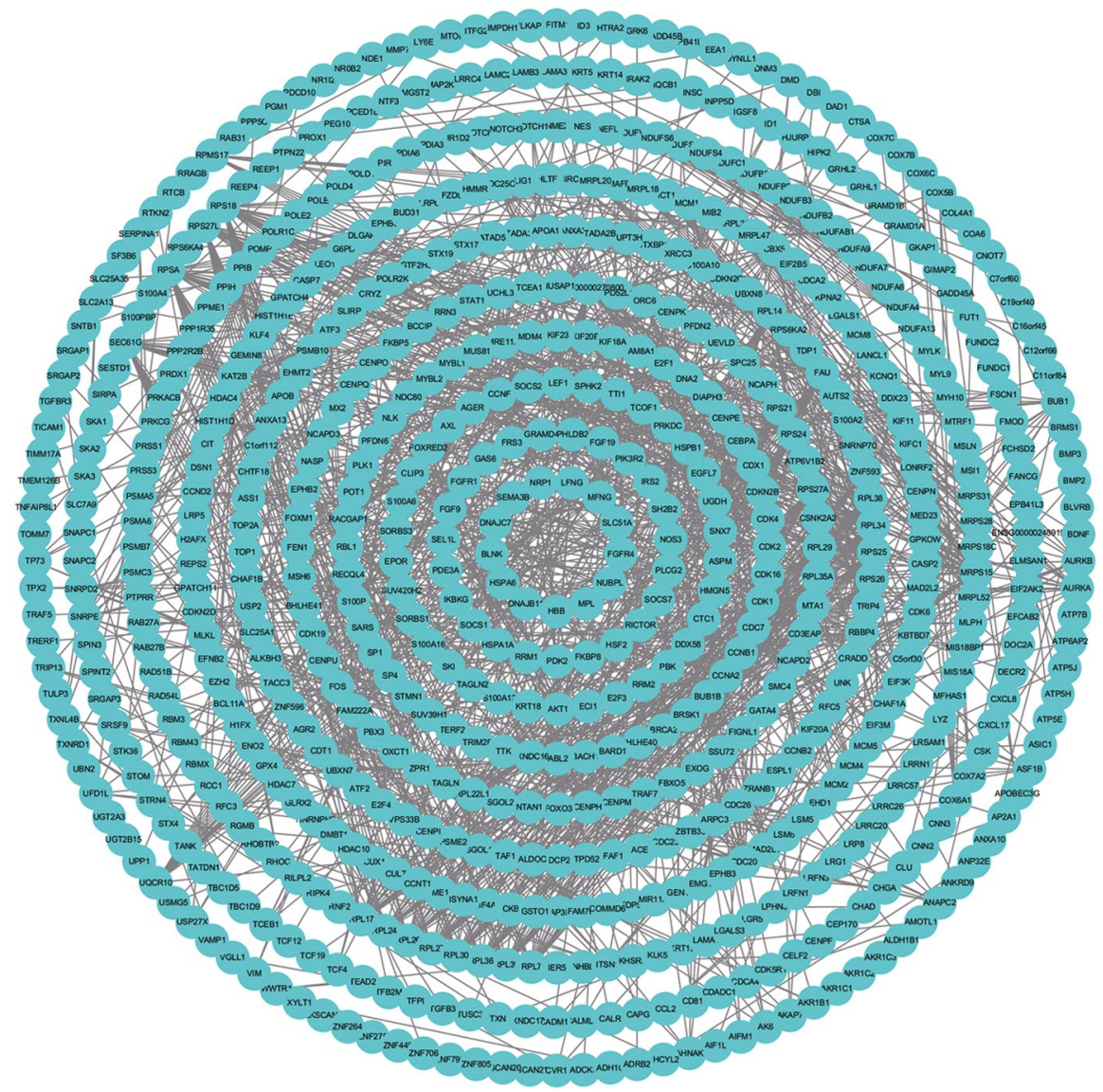

Fig. 10 Protein-protein interaction (PPI) networks of the 24-h group

of RRM2 and KIF20A usually accompanies the occurrence of cancer and plays a malignant role in cancer (Kitab et al. 2019; Morikawa et al. 2010; Sheng et al. 2018; Xiong et al. 2019).

In the results of this study, the down-regulation of NOTCH1 expression and the up-regulation of RRM2 and KIF20A provide a feasible direction for subsequent SARS-CoV-2 research. CCNA2 is the cyclin A2 gene, which is the gene encoding cyclin A2 on human chromosome 4 (Pagano et al. 1992). When a virus infects a cell, its genetic information can activate CCNA2 and promote the cell cycle. In addition, the overexpression of CCNA2 can enhance the reproduction, metastasis, and invasive ability of cancer cells and is closely connected to the occurrence and deterioration of ovarian cancer, liver cancer, and esophageal squamous cell carcinoma (ESCC) (Ruan et al. 2017). TTK, also known as MPS1, is a protein kinase. The up-regulation of TTK can activate $\mathrm{PKCa} / \mathrm{ERK} 1 / 2$ to promote the division of colon cancer cells and the lack of TTK can lead to apoptosis (Zhang et al. 2017, 2019; 


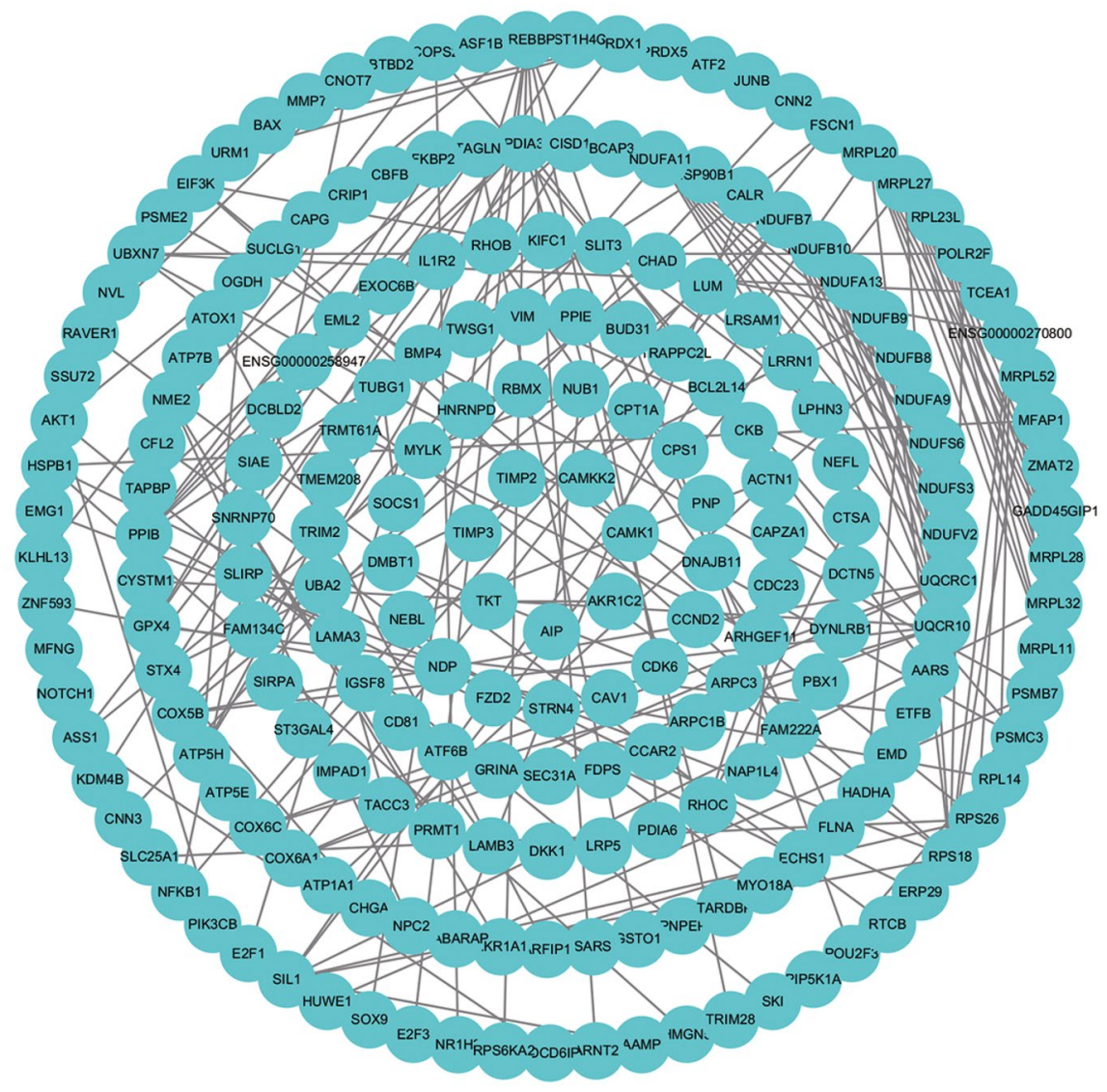

Fig. 11 Protein-protein interaction (PPI) networks of the 60-h group

Kaistha et al. 2014). The protein expressed by BUB1B is the BUB1 mitotic checkpoint serine/threonine kinase $\beta$, which plays an important part in the inspection of the spindle during mitosis. Furthermore, the expression of BUB1B in colon cancer tissues is higher than in normal colon tissues (Burum-Auensen et al. 2007). PLK1 is a member of the Polo-like family of mammals. It is located on the centrosome during mitosis and is usually overexpressed in cancer cells (Malumbres and Barbacid 2007). Moreover, PLK1 is also highly expressed in certain kinds of cancer, such as esophageal cancer and gastric cancer (Takahashi et al. 2003; Song et al. 2018). The TTK, BUB1B, PLK1, and CCNA2 genes and their translation products all perform significant tasks in the normal cell division cycle. During SARS-CoV-2 infection, the up-regulation of these four genes not only provides favorable conditions for the spread of the virus but also hints at the possibility of chromosomal abnormalities and other genetic material damage in the host cells. This result corresponds to the 


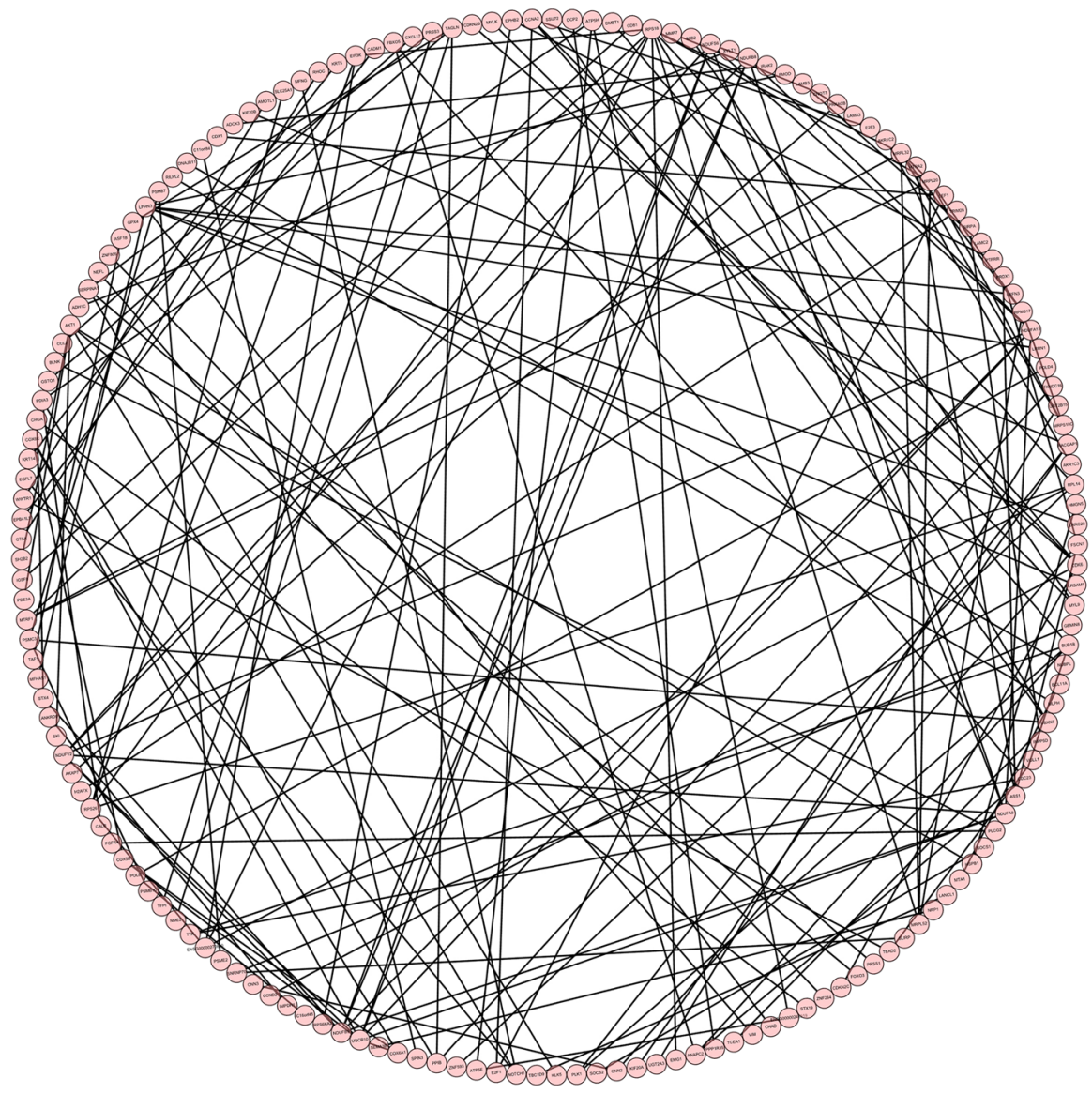

Fig. 12 PPI network of the intersection between 24- and 60-h groups

aforementioned expression results of NOTCH1, RRM2, and KIF20A. Therefore, the results of this study can provide a certain direction and basis for subsequent researchers for exploring the relationship between SARS-CoV-2 and cancer.

In conclusion, the nine hub genes obtained through statistical tests in this experiment all play an indispensable role in cell growth, reproduction, and disease. Therefore, this study analyzed their differential expression during the SARS-CoV-2 infection process, aiming to understand the subsequent diseases that SARS-CoV-2 may induce so as to facilitate their timely prevention. However, this study has certain limitations. For instance, the sample size of the experimental data could be further expanded, and the intestinal organs could not perfectly simulate the human environment. 


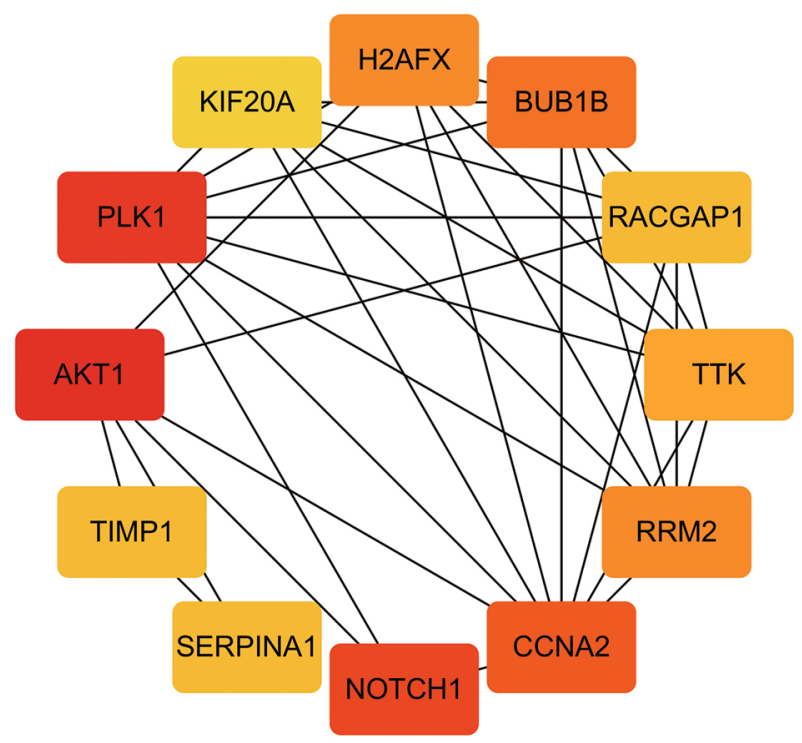

Fig. 13 PPI network of the top twelve genes
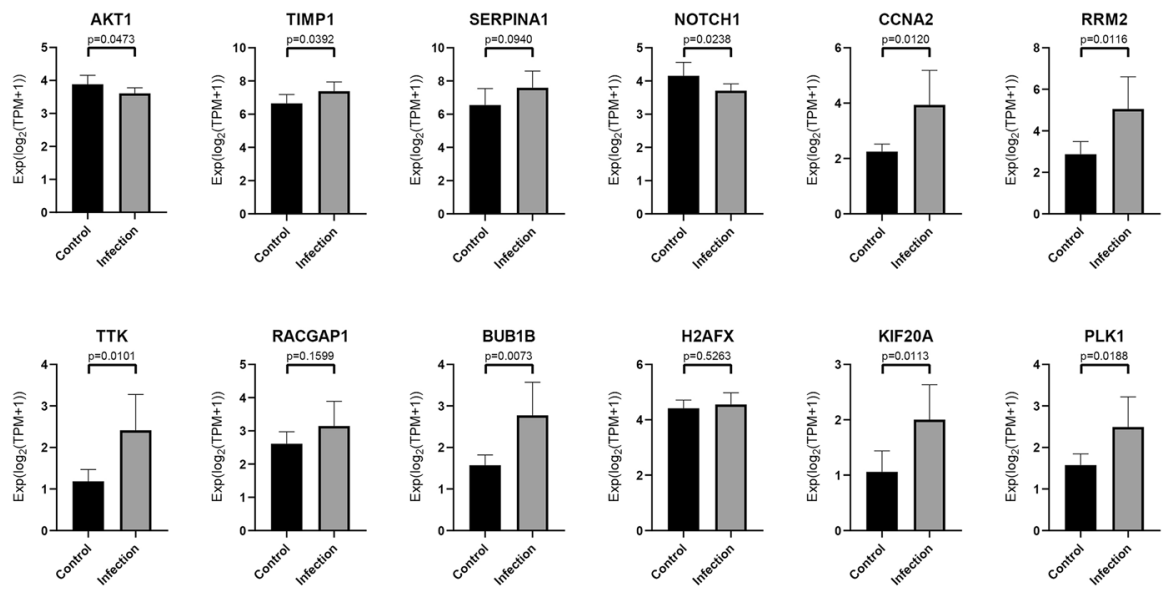

Fig. $14 \mathrm{t}$ tests and non-parametric tests of hub genes

Supplementary Information The online version contains supplementary material available at https://doi. org/10.1007/s10528-021-10144-w.

Open Access This article is licensed under a Creative Commons Attribution 4.0 International License, which permits use, sharing, adaptation, distribution and reproduction in any medium or format, as long as you give appropriate credit to the original author(s) and the source, provide a link to the Creative Commons licence, and indicate if changes were made. The images or other third party material in this article are included in the article's Creative Commons licence, unless indicated otherwise in a credit line to the 
material. If material is not included in the article's Creative Commons licence and your intended use is not permitted by statutory regulation or exceeds the permitted use, you will need to obtain permission directly from the copyright holder. To view a copy of this licence, visit http://creativecommons.org/licen ses/by/4.0/.

\section{References}

Arranz A, Doxaki C, Vergadi E, Martinez de la Torre Y, Vaporidi K, Lagoudaki ED, Ieronymaki E, Androulidaki A, Venihaki M, Margioris AN et al (2012) Akt1 and Akt2 protein kinases differentially contribute to macrophage polarization. Proc Natl Acad Sci USA 109:9517-9522

Burum-Auensen E, Deangelis PM, Schjølberg AR, Røislien J, Andersen SN, Clausen OP (2007) Spindle proteins Aurora A and BUB1B, but not Mad2, are aberrantly expressed in dysplastic mucosa of patients with longstanding ulcerative colitis. J Clin Pathol 60:1403-1408

Chan JF, Yuan S, Kok KH, To KK, Chu H, Yang J, Xing F, Liu J, Yip CC, Poon RW et al (2020) A familial cluster of pneumonia associated with the 2019 novel coronavirus indicating person-to-person transmission: a study of a family cluster. Lancet 395:514-523

Cipriano M, Ruberti E, Giacalone A (2020) Gastrointestinal infection could be new focus for coronavirus diagnosis. Cureus 12:e7422

Geller A, Yan J (2020) Could the induction of trained immunity by $\beta$-glucan serve as a defense against COVID-19? Front Immunol 11:1782

Gene Ontology Consortium (2006) The gene ontology (GO) project in 2006. Nucleic Acids Res 34:D322-326

Hajifathalian K, Mahadev S, Schwartz RE, Shah S, Sampath K, Schnoll-Sussman F, Brown RS Jr, CarrLocke D, Cohen DE, Sharaiha RZ (2020) SARS-COV-2 infection (coronavirus disease 2019) for the gastrointestinal consultant. World J Gastroenterol 26:1546-1553

Han C, Duan C, Zhang S, Spiegel B, Shi H, Wang W, Zhang L, Lin R, Liu J, Ding Z, Hou X (2020) Digestive symptoms in COVID-19 patients with mild disease severity: clinical presentation, stool viral RNA testing, and outcomes. Am J Gastroenterol 115:916-923

Harapan H, Itoh N, Yufika A, Winardi W, Keam S, Te H, Megawati D, Hayati Z, Wagner AL, Mudatsir M (2020) Coronavirus disease 2019 (COVID-19): a literature review. J Infect Public Health 13:667-673

He J, Tao H, Yan Y, Huang SY, Xiao Y (2020) Molecular mechanism of evolution and human infection with SARS-CoV-2. Viruses 12:428

Huang C, Wang Y, Li X, Ren L, Zhao J, Hu Y, Zhang L, Fan G, Xu J, Gu X et al (2020) Clinical features of patients infected with 2019 novel coronavirus in Wuhan, China. Lancet 395:497-506

Jin X, Lian JS, Hu JH, Gao J, Zheng L, Zhang YM, Hao SR, Jia HY, Cai H, Zhang XL et al (2020) Epidemiological, clinical and virological characteristics of 74 cases of coronavirus-infected disease 2019 (COVID-19) with gastrointestinal symptoms. Gut 69:1002-1009

Kaistha BP, Honstein T, Müller V, Bielak S, Sauer M, Kreider R, Fassan M, Scarpa A, Schmees C, Volkmer H et al (2014) Key role of dual specificity kinase TTK in proliferation and survival of pancreatic cancer cells. Br J Cancer 111:1780-1787

Kanehisa M, Goto S (2000) KEGG: kyoto encyclopedia of genes and genomes. Nucleic Acids Res 28:27-30

Kitab B, Satoh M, Ohmori Y, Munakata T, Sudoh M, Kohara M, Tsukiyama-Kohara K (2019) Ribonucleotide reductase $\mathrm{M} 2$ promotes RNA replication of hepatitis C virus by protecting NS5B protein from hPLIC1-dependent proteasomal degradation. J Biol Chem 294:5759-5773

Lamers MM, Beumer J, van der Vaart J, Knoops K, Puschhof J, Breugem TI, Ravelli RBG, Paulvan Schayck J, Mykytyn AZ, Duimel HQ, van Donselaar E, Riesebosch S, Kuijpers HJH, Schipper D, Van de Wetering WJ, Degraaf M, Koopmans M, Cuppen E, Peters PJ, Haagmans BL, Clevers H (2020) SARS-CoV-2 productively infects human gut enterocytes. Science 369(6499):50-54. https://doi.org/10.1126/science.abc1669 
Li LQ, Huang T, Wang YQ, Wang ZP, Liang Y, Huang TB, Zhang HY, Sun W, Wang Y (2020) COVID-19 patients' clinical characteristics, discharge rate, and fatality rate of meta-analysis. J Med Virol 92:577-583

Liang B, Li C, Zhao J (2016) Identification of key pathways and genes in colorectal cancer using bioinformatics analysis. Med Oncol 33:111

Malumbres M, Barbacid M (2007) Cell cycle kinases in cancer. Curr Opin Genet Dev 17:60-65

Morikawa T, Hino R, Uozaki H, Maeda D, Ushiku T, Shinozaki A, Sakatani T, Fukayama M (2010) Expression of ribonucleotide reductase M2 subunit in gastric cancer and effects of RRM2 inhibition in vitro. Hum Pathol 41:1742-1748

Ostaszewski M, Mazein A, Gillespie ME, Kuperstein I, Niarakis A, Hermjakob H, Pico AR, Willighagen EL, Evelo CT, Hasenauer J, Schreiber F, Dräger A, Demir E, Wolkenhauer O, Furlong LI, Barillot E, Dopazo J, Orta-Resendiz A, Messina F, Valencia A, Funahashi A, Kitano H, Auffray C, Balling R, Schneider R (2020) COVID-19 Disease Map, building a computational repository of SARS-CoV-2 virus-host interaction mechanisms. Sci Data 7(1):136. https://doi.org/10.1038/ s41597-020-0477-8

Pagano M, Pepperkok R, Verde F, Ansorge W, Draetta G (1992) Cyclin A is required at two points in the human cell cycle. EMBO J 11:961-971

Portincasa P, Krawczyk M, Smyk W, Lammert F, Di Ciaula A (2020) COVID-19 and non-alcoholic fatty liver disease: two intersecting pandemics. Eur J Clin Invest 50:e13338

Prins GH, Olinga P (2020) Potential implications of COVID-19 in non-alcoholic fatty liver disease. Liver Int 40(10):2568

Ruan JS, Zhou H, Yang L, Wang L, Jiang ZS, Wang SM (2017) CCNA2 facilitates epithelial-to-mesenchymal transition via the integrin $\alpha v \beta 3$ signaling in NSCLC. Int J Clin Exp Pathol 10:8324-8333

Safari Z, Gérard P (2019) The links between the gut microbiome and non-alcoholic fatty liver disease (NAFLD). Cell Mol Life Sci 76:1541-1558

Sheng Y, Wang W, Hong B, Jiang X, Sun R, Yan Q, Zhang S, Lu M, Wang S, Zhang Z et al (2018) Upregulation of KIF20A correlates with poor prognosis in gastric cancer. Cancer Manag Res 10:6205-6216

Song G, Xu S, Zhang H, Wang Y, Xiao C, Jiang T, Wu L, Zhang T, Sun X, Zhong L et al (2016) TIMP1 is a prognostic marker for the progression and metastasis of colon cancer through FAK-PI3K/AKT and MAPK pathway. J Exp Clin Cancer Res 35:148

Song R, Hou G, Yang J, Yuan J, Wang C, Chai T, Liu Z (2018) Effects of PLK1 on proliferation, invasion and metastasis of gastric cancer cells through epithelial-mesenchymal transition. Oncol Lett 16:5739-5744

Takahashi T, Sano B, Nagata T, Kato H, Sugiyama Y, Kunieda K, Kimura M, Okano Y, Saji S (2003) Polo-like kinase 1 (PLK1) is overexpressed in primary colorectal cancers. Cancer Sci 94:148-152

Villapol S (2020) Gastrointestinal symptoms associated with COVID-19: impact on the gut microbiome. Transl Res 226:57-69

Wang Y, Wang Y, Chen Y, Qin Q (2020) Unique epidemiological and clinical features of the emerging 2019 novel coronavirus pneumonia (COVID-19) implicate special control measures. J Med Virol 92:568-576

Xie S, Jiang X, Zhang J, Xie S, Hua Y, Wang R, Yang Y (2019) Identification of significant gene and pathways involved in HBV-related hepatocellular carcinoma by bioinformatics analysis. PeerJ 30(7):e7408. https://doi.org/10.7717/peerj.7408

Xiong M, Zhuang K, Luo Y, Lai Q, Luo X, Fang Y, Zhang Y, Li A, Liu S (2019) KIF20A promotes cellular malignant behavior and enhances resistance to chemotherapy in colorectal cancer through regulation of the JAK/STAT3 signaling pathway. Aging (albany NY) 11:11905-11921

Zhang XL, Han WX, Ma YM, Baohan HW, Zhao XH, Wang HY (2017) Expression of tyrosine and threonine protein kinase in carcinogenic process of colorectal cancer and its relationship with prognosis. Zhonghua Zhong Liu Za Zhi 39:172-177

Zhang L, Jiang B, Zhu N, Tao M, Jun Y, Chen X, Wang Q, Luo C (2019) Mitotic checkpoint kinase Mps1/TTK predicts prognosis of colon cancer patients and regulates tumor proliferation and differentiation via PKC $\alpha / E R K 1 / 2$ and PI3K/Akt pathway. Med Oncol 37:5

Zhou J, Marten NW, Bergmann CC, Macklin WB, Hinton DR, Stohlman SA (2005) Expression of matrix metalloproteinases and their tissue inhibitor during viral encephalitis. J Virol 79:4764-4773

Zhu N, Zhang D, Wang W, Li X, Yang B, Song J, Zhao X, Huang B, Shi W, Lu R et al (2020) A novel coronavirus from patients with pneumonia in China, 2019. N Engl J Med 382:727-733 
Zuwała K, Golda A, Kabala W, Burmistrz M, Zdzalik M, Nowak P, Kedracka-Krok S, Zarebski M, Dobrucki J, Florek D et al (2015) The nucleocapsid protein of human coronavirus NL63. PLoS ONE 10:e117833

Publisher's Note Springer Nature remains neutral with regard to jurisdictional claims in published maps and institutional affiliations. 